procedure related or associated deaths being rare. GI cancers are twice as likely to be associated than non-GI cancers.

Disclosure of Interest None Declared.

\section{PWE-057 STENT PLACEMENT IN PALLIATIVE OESOPHAGO- GASTRIC CANCER: CHANGED PRACTICE WITH IMPROVED OUTCOMES}

doi:10.1136/gutjnl-2013-304907.346

1," M Kasi, ${ }^{2} \mathrm{~S}$ Beg, ${ }^{2} \mathrm{~S}$ Sargeant, ${ }^{2} \mathrm{D}$ Morris. 'Gastroenterology, Derriford Hospital, Plymouth NHS Trust, Plymouth; ' ${ }^{2}$ Gastroenterology, East and North Hertfordshire NHS Trust, Hertfordshire, UK

Introduction Oesophageal cancer is often diagnosed late in its pathological process and as a consequence management is often focussed on palliation of symptoms. The insertion of oesophageal stents tend to occur in small numbers and as such any individual endoscopist will perform only a few in a given time period. In this study we aim to establish whether by limiting this procedure to a few operators we can improve outcomes by increasing operator experience.

Methods This is a retrospective review of palliative stenting in patients with advanced oesophageal and oesophagogastric cancers across East and North Hertfordshire NHS Trust in the 15 month period from $1^{\text {st }}$ April $2011-31^{\text {st }}$ July 2012. We audited endoscopy reports and our prospectively maintained Upper Gastrointestinal Cancer database for any reported post procedural complications and calculated 7,14 and 30 day mortality rate for these cohort patients. We also re-audited complications following stent insertion from March 2010-2011 where stents were performed by the first available gastroenterologists. Results were analysed according to BSG Quality indicators and compared with National Oesophagogastric cancer Audit 2010.

Results 20 patients had palliative stents with in this time period. The median age was 74 and male to female ratio is $3: 2.70 \%$ of cases were adenocarcinoma and $20 \%$ were Squamous cell carcinoma. The combination of pharyngeal anaesthesia and sedation were used only in $10 \%(n=2)$ compared to $21 \%$ last year. Procedures carried under fluoroscopy guidance were $100 \%$ compared to $36 \%$ nationally. Boston Scientific Ultraflex covered metal stents were used in $95 \%$ of patients. All the stents were deployed successfully. No reported complications of stent migration (compared to $12 \%$ migration rates last year), perforation and haemorrhage post procedure. This was achieved with two gastroenterologists with special interest performing the procedure compared to five consultants last year. Our 7, 14 and 30 day mortality are as shown in the graph below.

Conclusion We use laser therapy first line where appropriate. This usually achieves a better dysphagia grade than stenting initially. This means that our patients have been palliated for several months before stents are inserted. Despite this patient selection seems to be appropriate as most survived more than 30 days. No complications were noted with insertion and post stent, this was a major improvement from last year's audit. From this study we have demonstrated that by treating oesophageal stent insertion as a specialist procedure, with dedicated operators we are able to minimise complication rates.

Disclosure of Interest None Declared.

\section{PWE-058 TISSUE ACQUISITION FROM SOLID PANCREATIC LESIONS - ENDOSCOPY OR SURGERY}

doi:10.1136/gutjnl-2013-304907.347

1." M Nayar, 'D Tai Kung, 'B Haugk, 'S White, 'D Manas, 'B Jaques, 'G Sen, 'J French, ${ }^{1} \mathrm{R}$ Charnley, ${ }^{1} \mathrm{~K}$ Oppong. ' $H P B$ Unit, Freeman Hospital, Newcastle Upon Tyne, UK

Introduction The accurate diagnosis of solid pancreatic masses is important in directing appropriate management of patients. The methods commonly used are percutaneous, laparoscopic and EUS guided biopsy of these lesions. Laparoscopic guided biopsy consumes theatre time and space and can be more expensive than the alternatives. In our unit; laparoscopic guided biopsy is reserved for patients who have an inconclusive result from an EUS guided biopsy or are considered for trial resection. The aim of this study was to look at the diagnostic performance of endoscopic ultrasound (EUS) guided biopsy (fine needle aspiration (FNA) or pro-core biopsy) and laparoscopic (lap) guided biopsy of solid pancreatic masses in a large HPB referral centre.

Methods Retrospective review of patients undergoing EUS or laparoscopic guided biopsy for solid lesions between January 2011 and March 2012. Data was obtained from a dedicated prospectively maintained database in the histopathology department. Final diagnosis was based on positive histology/cytology of pancreatic adenocarcinoma. Benign cases were followed up for a period of at least six months.

Results 464 specimens from the pancreas (histology + cytology including pancreatic resections) were received by the histopathology department during this period. Of these 275/464 (59\%) patients had tissue biopsy of solid lesions.

These included: EUS guided biopsy $=253$ and Laparoscopic guided biopsy $=22$.

In the latter group; $10 / 22(45 \%)$ had a previous EUS of which $8 / 10$ had an accurate diagnosis. $12 / 22(54 \%)$ patients went straight for laparoscopic guided biopsy. For the purposes of this study; highly suspicious and malignant samples were categorised as malignant. The accuracy, sensitivity and negative predictive value for EUS guided biopsy and laparoscopic guided biopsy were $92 \% / 96 \% ; 90 \% / 94 \%$ and $74 \% / 89 \%$ respectively. The inadequate aspirate rate was $5 \%$ and $0 \%$ respectively. There was no significant difference between the two groups. The cost of performing these procedures in our trust were: EUS guided biopsy £1094 and lap guided biopsy $£ 2164$.

Conclusion In our unit;EUS guided biopsy of solid pancreaticobiliary lesions provides high diagnostic accuracy with a low inadequate aspirate rate. Our data supports the role of EUS guided FNA as the first modality of tissue acquisition from the pancreas. Though this data also shows the cost effectiveness of EUS guided biopsy over lap guide biopsy; in units with lower diagnostic accuracy of EUS guided biopsy the cost benefit may not be realised.

Disclosure of Interest None Declared.

\section{PWE-059 SINGLE DOSE ENDOSCOPIC THROMBIN INJECTION FOR ACUTE VARICEAL BLEEDING}

doi:10.1136/gutjnl-2013-304907.348

1."M Smith, 'M Widlak, 'N Fisher, 'S Ishaq. 'Dudley Group of Hospitals NHS Foundation Trust, NHS, Dudley, UK

Introduction Endoscopic Human Thrombin injection appears a technically simple yet efficacious alternative to cyanoacrylate for gastric varices with fewer complications from studies to date, but data remains limited. We evaluated the outcomes of patients following single thrombin injection treatment for acute bleeding from oesophageal and gastric varices.

Methods Retrospective review of patients receiving endoscopic human thrombin injection (Tisseel Baxter Intl Inc.) for active bleeding from varices at a UK centre 2011-2012.

Results 15 patients (67\% male, mean age 56 (SD 10)), received human thrombin injection for actively bleeding varices. Mechanism of portal hypertension was alcoholic cirrhosis in 12 patients $(80 \%)$, extra-hepatic in 3 (20\%). Extrahepatic portal hypertension was due to cancer, portal vein thrombosis and splenic vein thrombosis respectively. Mean MELD was 15 (SD 6). Childs grade was $\mathrm{A}, \mathrm{B}, \mathrm{C}$ in $6 \%, 47 \%$ and $47 \%$ respectively. Bleeding varices were identified as gastric in 13 patients (87\%), oesophageal in $2(13 \%)$. These 2 cases were not amenable to further banding due to band-induced fibrosis. 